\title{
CONTEXTOS LOCALES E INTERNACIONALES DE PUBLICACIÓN CIENTÍFICA: ANÁLISIS RETÓRICO-DISCURSIVO DE LOS RESÚMENES DE ARTÍCULOS DE INVESTIGACIÓN EN INGLÉS EN EL ÁMBITO DE LA SOCIOLOGÍA
}

\author{
INTERNATIONAL AND LOCAL CONTEXTS OF SCIENTIFIC PUBLICATION: \\ RHETORICAL-DISCURSIVE ANALYSIS OF SOCIOLOGY ABSTRACTS IN ENGLISH
}

\author{
ROSA LORÉs SANZ \\ Universidad de Zaragoza \\ rlores@unizar.es \\ Silvia Murillo Ornat \\ Universidad de Zaragoza \\ smurillo@unizar.es
}

Enviado: 01/03/2018

Aceptado: 05/10/2018

\section{Resumen}

Los abstracts desempeñan un importante papel como herramientas que permiten ahorrar tiempo y gestionar la información. Muchas revistas académicas nacionales no anglófonas requieren que los autores redacten una versión en inglés de estas secciones. En este artículo nos proponemos identificar y analizar los patrones retóricos y léxico-gramaticales (fraseología) que caracterizan a los abstracts escritos en español y traducidos al inglés, publicados

\begin{abstract}
Abstracts play a major role as time-saving and information-managing devices. Many nonAnglophone national journals require authors to write an English version of the abstracts. In this paper our aim is to identify and analyze the rhetorical and lexicogrammatical patterns (phraseology) which characterize abstracts written in Spanish and translated into English, published in national (Spanish) journals. These texts are contrastively explored with abstracts
\end{abstract}

\footnotetext{
* Este trabajo es una contribución al proyecto "Hacia una mayor visibilidad y diseminación de la investigación científica: estudio lingüístico, retórico y pragmático de géneros digitales en inglés como lengua internacional" (FFI2017-84205), financiado por el Ministerio de Economía, Industria y Competitividad.

Para citar este artículo / To cite this article: Lorés Sanz, Rosa y Silvia Murillo Ornat (2018). Contextos locales e internacionales de publicación científica: análisis retórico-discursivo de los resúmenes de artículos de investigación en inglés en el ámbito de la sociología. ELUA, 32: 331-348. doi: 10.14198/ELUA2018.32.15
}

Enlace / Link: http://dx.doi.org/10.14198/ELUA2018.32.15 
en revistas nacionales (españolas). Estos textos se comparan con abstracts escritos en inglés como lengua franca (ILF) y publicados en revistas internacionales. Para tales fines, se consideran 30 abstracts de cada contexto linguacultural. La disciplina elegida es la Sociología, un campo en el que los investigadores españoles han comenzado a publicar recientemente a nivel internacional. Los resultados muestran que los abstracts traducidos al inglés presentan diversas similitudes y diferencias. Las diferencias pueden interpretarse como el resultado de la influencia de ciertos patrones de la escritura académica en español.

PALABRAS CLAVE: abstracts, inglés como lengua franca, traducción, estructura retórica, fraseología. written in English as a Lingua Franca (ELF) and published in international journals. For such purposes, 30 texts of each linguacultural context are considered. The discipline chosen is Sociology, quite a recent field for Spanish academics in terms of international publications. The results show that Spanish abstracts in English show features of similarity and difference as regards their rhetorical structure and its realization in terms of lexicogrammar. Divergences may be interpreted as the result of the influence of certain patterns in Spanish academic writing.

KEYWORDS: abstracts, English as a lingua franca, translation, rhetorical structure, phraseology.

\section{INTRODUCCIÓN}

En el actual contexto de investigación científica, el flujo de publicaciones está alcanzando máximos nunca antes conocidos. En estos momentos las principales bases de datos incluyen una ingente cantidad de revistas científicas a las que contribuyen un número creciente de académicos. El artículo de investigación constituye el género académico por excelencia, que permite dar voz al investigador y dar forma a su producción científica. El incremento exponencial del volumen de artículos de investigación publicados está potenciando el interés no solo por este género académico imprescindible sino, además, por otro género académico muy relacionado con el anterior, hasta el punto de ser considerado una sección del mismo, pero que cuenta con una entidad propia: el resumen del artículo de investigación o abstract. El significativo papel que juega el abstract en nuestro actual panorama investigador viene determinado precisamente por el ya inmanejable número de publicaciones que día a día ven la luz en las miles de revistas científicas que se publican en las distintas áreas del saber. El abstract se ha convertido en una eficaz herramienta (junto con otras manifestaciones textuales como las palabras clave o los highlights) que facilita la navegación primero, y el filtrado y selección después, de aquellas publicaciones que puedan ser de interés para el investigador, quien necesita nutrirse de la producción de otros para seguir avanzando en el conocimiento de su disciplina.

Junto a este panorama de creciente (y prácticamente inmanejable) volumen de información científica que se está dibujando en nuestros días, y quizás también a consecuencia del mismo, se constata el papel tan significativo que ha adquirido la lengua inglesa como lengua de comunicación en el mundo científico y académico global. Sin entrar a valorar aquí las razones, de tipo económico, histórico y social, que han encumbrado al inglés como lengua de comunicación científica internacional, lo cierto es que, aunque no se puede afirmar que toda la ciencia se expresa en inglés, sí lo hace una buena parte de ella, especialmente por lo que respecta a ciertas áreas disciplinares, especialmente en el ámbito de las ciencias puras, y a ciertos contextos lingüístico-culturales, como es el norte de Europa. Siendo innegable el beneficio que el uso de una lengua franca en el ámbito científico pueda proporcionar al avance de la ciencia en general, ese 
mismo papel preponderante puede generar una serie de implicaciones no tan positivas, como el hecho de que algunas disciplinas estén relegando el uso de las lenguas nacionales y primando la expresión de la producción científica en lengua inglesa. Esta tendencia puede dar lugar a lo que Bennett (2014) denomina "epistemicidio", privando de esta manera a ciertas áreas de conocimiento de aproximaciones al objeto de estudio propias del contexto lingüístico y cultural nacional, que bien podrían contribuir al desarrollo y avance de la disciplina (Seidlhofer 2012; Bennett 2014). Son, por tanto, fundamentalmente, aquellos para los que el inglés no es su lengua nativa los que de manera más directa son conscientes del impacto que el uso transnacional de la lengua inglesa está teniendo en el ámbito científico. Según estiman muchos estudiosos del tema (ver p. ej. Canagarajah 1996, 2002; Tardy 2004; Ferguson 2007; Flowerdew 2007, 2008), esta situación de falta de igualdad de oportunidades coloca a los investigadores no anglófonos en una posición poco ventajosa, al optar por la publicación de sus trabajos en una lengua que no es la suya, o al verse obligados a hacerlo por unas $u$ otras razones.

Las dificultades de los no nativos en la composición y redacción de textos académicos ha sido frecuente objeto de estudios interculturales, al menos desde la publicación y consiguiente impacto del famoso artículo de Robert Kaplan "Cultural thought patterns in intercultural education" (1966), que, de alguna manera, marcó el inicio de los estudios de retórica contrastiva. Dentro de estos estudios, y en el ámbito de la lengua para fines académicos, el artículo de investigación ha sido sin duda el principal foco de análisis, lo cual no ha impedido que numerosos trabajos, de manera creciente, se centraran en el estudio del abstract como género académico, incremento que ha ido produciéndose de manera paralela al cada vez más significativo papel del abstract en la gestión y producción de la investigación científica. Los estudios contrastivos de este género en inglés y en español han sido quizás los más numerosos (ver, entre otros, Martín-Martín 2003, 2005; Divasson y León Pérez 2006; Lorés-Sanz 2006, 2009a, 2014; Bellés-Fortuño y Querol-Julián 2010; Burgess y Martín-Martín 2010; Alonso-Almeida 2014), aunque son varias las lenguas de contraste que se han utilizado en los estudios de este género académico: alemán (Busch-Lauer 1995, 2014), árabe (Alharbi 1997; Alharbi y Swales 2011), francés (van Bonn y Swales 2007), italiano (Diani 2014) y turco (Işsk-Taş 2018), entre otras.

Como una evolución natural de los estudios de retórica contrastiva en los que el permanente parámetro de contraste es la lengua inglesa, el estudio del inglés como lengua franca (ILF) ha dado lugar, desde hace poco más de una década, a importantes aportaciones al estudio de la lengua inglesa, sobre todo en lo que a posicionamiento frente a la imposición del inglés como lengua nativa se refiere. Así, el ILF, que se define de forma consensuada como una lengua común utilizada por hablantes que no comparten su lengua nativa, ha sido explorado desde distintos niveles, como el fonológico (Jenkins 2000) y el léxico-gramatical (Cogo y Dewey 2012), así como el nivel pragmático (House 2009; Mauranen 2009). Aunque la interacción oral ha recibido, con mucho, el mayor foco de atención por parte de los estudiosos del ILF, los textos académicos escritos comienzan a ser también objeto de estudio, como demuestra la publicación de recientes trabajos (p. ej. Carey 2013; Mur-Dueñas 2013, 2015, 2016, 2018; Lorés-Sanz 2016, 2018a, 2018b, en prensa; Murillo 2018). Otro indicador de la creciente atención que el lenguaje académico escrito está recibiendo es la compilación del corpus $\mathrm{SciELF}^{1}$, de artículos de investigación

1 SciELF 2015. The SciELF Corpus. Director: Anna Mauranen. http://www.helsinki.fi/elfa/scielf.html (1/3/2018). 
no editados redactados en inglés por investigadores de diversas L1, coordinado desde la Universidad de Helsinki (Finlandia), así como los estudios que de él están emanando (Lafuente-Millán en prensa; Mur-Dueñas en prensa; Murillo en prensa).

Por otra parte, junto a la necesidad creciente de publicar en revistas internacionales en inglés, impulsada en gran medida por los sistemas de calidad de las instituciones académicas y organismos de investigación para medir la producción científica, se observa la cada vez más frecuente presión sobre las revistas nacionales para utilizar la lengua inglesa en sus publicaciones de una manera u otra, lo que, entre otras cosas, les facilita un sello de calidad y su inclusión en bases de datos internacionales. Esto redunda, primero, en su capacidad para atraer un mayor número de propuestas y, seguidamente, en la difusión, a mayor escala, de la producción científica local. Una de las maneras más habituales en las que las revistas cumplen con estos objetivos es incluir una versión en inglés de ciertas partes del artículo de investigación, lo que de manera habitual supone contar con un resumen del artículo de investigación en esta lengua. Así, en el contexto español, la Guía de evaluación de la quinta convocatoria de evaluación de la calidad editorial y científica de las revistas científicas españolas ${ }^{2}$ (2015) de la FECYT ${ }^{3}$ incluye el cumplimiento obligatorio de 12 indicadores de calidad, entre los que se encuentra el siguiente:

“6. Traducción de los títulos de los artículos, palabras clave y resúmenes al inglés: todos los artículos científicos de la revista deberán ofrecer, al menos, el título, resumen y palabras clave en inglés".

Se constata, por tanto, que la traducción de los resúmenes de investigación es demandada por las publicaciones de ámbito nacional y merece, consiguientemente, convertirse en objeto de estudio en tanto en cuanto puede constituir la puerta de acceso de la investigación local a la difusión internacional. Sin embargo, frente al numeroso volumen de estudios contrastivos inglés/español existentes sobre el abstract que arriba señalábamos, son escasos los que se centran en las traducciones al inglés de abstracts en español que acompañan a artículos publicados en revistas nacionales (ver Fernández Antolín et alii 2006; López-Arroyo et alii 2007; Perales-Escudero y Swales 2011).

En este artículo nos hemos propuesto explorar las traducciones al inglés de estos resúmenes desde perspectivas retórico-discursivas de contraste con los textos originales en español de los que parten y con otros abstracts publicados en inglés como lengua franca en revistas internacionales. Con el fin de minimizar el impacto de las variables disciplinares y lingüístico-culturales, el estudio se centrará en una sola disciplina, la Sociología. Esta área de conocimiento ha estado tradicionalmente dominada por investigadores anglosajones, prueba de lo cual es el hecho de que las revistas más prestigiosas de esta disciplina se localizan en los EE.UU. y en el Reino Unido. La elección de esta área disciplinar como ámbito de estudio responde a la reciente y creciente contribución que los sociólogos españoles están realizando en el contexto internacional, frente a otras áreas, generalmente del ámbito de las ciencias puras, con una tradición más asentada de participación de los investigadores españoles en contextos científicos globales. Nuestro objetivo es estudiar cómo el abstract, un género tan significativo desde el punto de vista del acceso a la información, se construye

2 https://evaluacionarce.fecyt.es/doc/2015GuiaEval5Conv.pdf (1/3/2018).

3 Fundación Española para la Ciencia y la Tecnología, dependiente del Ministerio de Economía, Industria y Competitividad. 
retórica y discursivamente en inglés por parte de autores pertenecientes a la misma comunidad disciplinar, pero arraigados en diferentes contextos lingüístico-culturales. Se trata de identificar patrones de similitud y divergencia entre traducciones al inglés y textos originales en ILF, teniendo en cuenta que, al estar escritos en inglés, ambos tipos de textos pueden ser accesibles para el lector internacional. En última instancia, la identificación de patrones retóricos y fraseológicos propios en estas colecciones de textos puede tener también implicaciones de tipo pedagógico, tanto para el campo de la escritura académica en inglés como lengua franca como de la enseñanza de la traducción español-inglés en el ámbito académico.

\section{DESCRIPCIÓN DEL CORPUS Y METODOLOGÍA}

Los objetivos de estudio que aquí nos hemos propuesto requieren la compilación de colecciones de textos que nos permitan identificar patrones retórico-discursivos. Con este fin, hemos construido un corpus integrado por 90 resúmenes (unas 13000 palabras) que acompañan a artículos de investigación de los siguientes contextos lingüístico-culturales, todos ellos en la disciplina de la Sociología:

- 30 resúmenes en inglés como lengua franca (subcorpus ILF)

- 30 resúmenes en español como L1 (subcorpus ESP)

- 30 traducciones al inglés de los resúmenes en español (subcorpus TRAD)

Los resúmenes están extraídos de 3 revistas nacionales y 3 internacionales, a razón de 10 textos por revista. Los volúmenes de donde se extrajeron fueron publicados entre los años 2003-2010.

Los subcorpus ESP y TRAD fueron compilados con textos de las siguientes revistas:

- Papers. Revista de Sociología (Universidad Autónoma de Barcelona)

- Revista Española de Investigaciones Sociológicas (REIS) (Centro de Investigaciones Sociológicas)

- Revista Internacional de Sociología (RIS) (Instituto de Estudios Sociales Avanzados de Andalucía).

El subcorpus ILF fue extraído de las siguientes revistas:

- British Journal of Sociology (London School of Economics)

- AJS (American Journal of Sociology) (The University of Chicago Press)

- Social Science Research (Elsevier).

Según consta en las correspondientes páginas web, todas las revistas están indizadas e incluidas en bases de datos internacionales ${ }^{4}$. Las instrucciones para los autores incluyen información sobre la redacción de resúmenes, que se anexa en el Apéndice 1 del presente estudio.

4 Papers. Revista de Sociología (http://papers.uab.cat/ [1/3/2018]); Revista Española de Investigaciones Sociológicas (REIS) (www.reis.cis.es [1/3/2018]); Revista Internacional de Sociología (RIS) (http://revintsociologia. revistas.csic.es [1/3/2018]). British Journal of Sociology (http://onlinelibrary.wiley.com/journal/10.1111/ (ISSN)1468-4446 [1/3/2018]); American Journal of Sociology (AJS) (http://www.journals.uchicago.edu/journals/ajs/ about [1/3/2018]); Social Science Research (https:/www.journals.elsevier.com/social-science-research/ [1/3/2018]). 
Mientras que la selección de los textos que se incluyeron en los subcorpus ESP (y, por tanto, en TRAD) fue aleatoria, con un número constante de artículos por año, para la compilación del subcorpus ILF primó el criterio de representatividad de todas las afiliaciones sobre el criterio de proporcionalidad. Aun así, hay mayor representación de las áreas geográficas que más publican en lengua inglesa a nivel internacional, como son los Países Bajos, el norte de Europa, Israel y algunos países asiáticos.

Este corpus multilingüe se concibió como una combinación de un corpus comparable, en dos lenguas distintas (ILF y ESP), y de un corpus de textos originales y traducciones (ESP y TRAD). El diseño de este corpus permitió la interacción entre dos perspectivas de análisis, la Lingüística Contrastiva y los Estudios de Traducción, que, según indica Granger (2003: 25), "rely on the same type of data, use the same software tools and are partly interested in the same corpus-based applications, notably reference materials - dictionaries, grammars and teaching methods".

Como antes hemos mencionado, el estudio comparativo de los tres subcorpus se llevó a cabo en el nivel retórico y en el nivel discursivo ${ }^{5}$. La identificación de la estructura retórica se llevó a cabo en términos de movimientos (moves) (Swales 1990), siguiendo una perspectiva top-down (Pho 2008), que se basa en la interpretación del contenido del texto y en la identificación de la función comunicativa de cada movimiento. En línea con esta metodología, se aplicó el modelo de estructura retórica de resúmenes propuesto por Lewin (2010) para las Ciencias Sociales. Lewin (2010) propone una estructura retórica común a los resúmenes de los artículos de investigación y a los que constituyen propuestas de participación en congresos. Este modelo incluye seis movimientos:

- Relevance

- Aims

- Gap

- Method

- Results

- Conclusions

Todos los textos que conformaban los tres subcorpus fueron analizados y etiquetados de forma independiente por las autoras siguiendo este modelo. En un segundo paso se revisaron aquellos pocos casos en los que no hubo coincidencia, hasta que esta fue del $100 \%$. Así mismo, se aplicó la prueba de chi-cuadrado para comprobar si los resultados obtenidos eran estadísticamente significativos ${ }^{6}$.

La identificación de los movimientos de la estructura retórica facilitó la búsqueda de patrones fraseológicos, algunos de los cuales a su vez juegan un importante papel en dicha identificación. Este rol identificador permite interpretar de forma adecuada la función comunicativa que tienen los movimientos dentro del abstract. La búsqueda de estos patrones atendió a una metodología corpus-driven (Tognini-Bonelli 2001). Se trata de un proceso

5 Por estudio del nivel retórico entendemos aquí la identificación y análisis de la estructura de los textos en función de los propósitos comunicativos que se realizan en cada uno de los movimientos del texto. El análisis del nivel discursivo, por su parte, conlleva el estudio de los rasgos léxico-gramaticales que caracterizan al texto.

6 Se utilizó la calculadora en línea de Preacher (2001). Con un p-valor $<0,05$ (el nivel normalmente utilizado en trabajos de Lingüística), se considera que los resultados son estadísticamente significativos. 
deductivo en el que no se aplican afirmaciones teóricas previas a los datos obtenidos en el análisis del corpus, como sí ocurre en la metodología corpus-based, donde el corpus actúa como "a repository of examples to expound, test, or exemplify given theoretical statements" (Tognini-Bonelli 2001: 10).

Se trataba de establecer aspectos de divergencia entre el subcorpus ILF y el subcorpus TRAD, es decir, entre textos escritos en lengua inglesa independientemente de la L1 de sus autores o en inglés como resultado de un proceso de traducción a partir de textos en español. El subcorpus ESP se utilizó como corpus de referencia a la hora de interpretar las selecciones léxicas del corpus TRAD y las divergencias encontradas con respecto al subcorpus ILF. La identificación de las unidades fraseológicas que apuntaban a la función comunicativa de los distintos movimientos se llevó a cabo en los tres subcorpus a través de lecturas manuales. Seguidamente, se utilizó la herramienta electrónica de análisis de corpus AntConc ${ }^{7}$ para establecer las frecuencias de estas unidades fraseológicas.

A modo de información contextual de cara a la interpretación de datos, se llevó a cabo una encuesta a través de un cuestionario enviado a los autores españoles de los 30 textos que conformaban los subcorpus ESP/TRAD, con el propósito de extraer información sobre el proceso seguido para la traducción al inglés de sus resúmenes. Entre otras cuestiones se les preguntaba expresamente por la participación de traductores o revisores de estilo (literacy brokers, Lillis y Curry 2010) y si estos agentes eran (1) nativos expertos en la disciplina, (2) nativos no expertos en la disciplina, (3) no nativos expertos en la disciplina o (4) no nativos no expertos en la disciplina.

Se recibieron 16 respuestas al cuestionario ${ }^{8}$, lo que supone un $53 \%$. Este porcentaje nos permite observar tendencias sobre las prácticas de los investigadores españoles en el campo de la Sociología. Así, 15 de los 16 textos habían sido traducidos por el propio autor $\mathrm{y}$, de esos, nueve habían sido enviados directamente para su publicación a los editores. Otros cinco fueron revisados previamente por no nativos expertos en la disciplina y uno fue revisado por un experto nativo en la disciplina. Solo un texto había sido traducido por un traductor profesional.

De acuerdo con estos datos, los investigadores españoles en la disciplina de Sociología tienden a traducir ellos mismos al inglés sus resúmenes y no hacen uso de servicios de traducción. Recurren a revisores en algún caso, y estos tienden a ser expertos en la disciplina más que en la lengua, lo cual parece indicar el mayor valor que dan los autores al contenido sobre la forma. Además, el hecho de que la mayoría de los autores traduzcan sus textos ellos mismos y los envíen a los editores sin revisión apunta, por un lado, hacia un cierto nivel de autoconfianza en su conocimiento de la lengua y, por otro, hacia una escasa conciencia del relevante papel que tienen los resúmenes de artículos en la difusión de la producción científica a escala internacional. Estas asunciones pueden explicar la falta de cuidado apreciado en algunos casos en los niveles lingüísticos y retóricos de los textos estudiados.

Este estudio etnometodológico ha resultado de gran utilidad para interpretar algunos de los datos cuantitativos que se incluyen en la siguiente sección.

7 http://www.antlab.sci.waseda.ac.jp/software.html (1/3/2018).

8 En el caso de los artículos en coautoría, se contactó con todos los autores, pero, cuando se obtuvo más de una respuesta, solo se computó una vez. La distribución de respuestas por revista fue como sigue: 4 de autores de Papers, 7 de REIS y 5 de RIS. 


\section{RESULTADOS}

\subsection{Estructura retórica}

En primer lugar, los resultados del estudio de la estructura retórica basado en la propuesta de Lewin (2010) aparecen reflejados en la Figura 1. Los resultados de los subcorpus ESP y TRAD están consignados conjuntamente, pues no se observó ninguna modificación en la estructura retórica de ningún abstract en el paso de la lengua origen (ESP) a la lengua meta (TRAD):

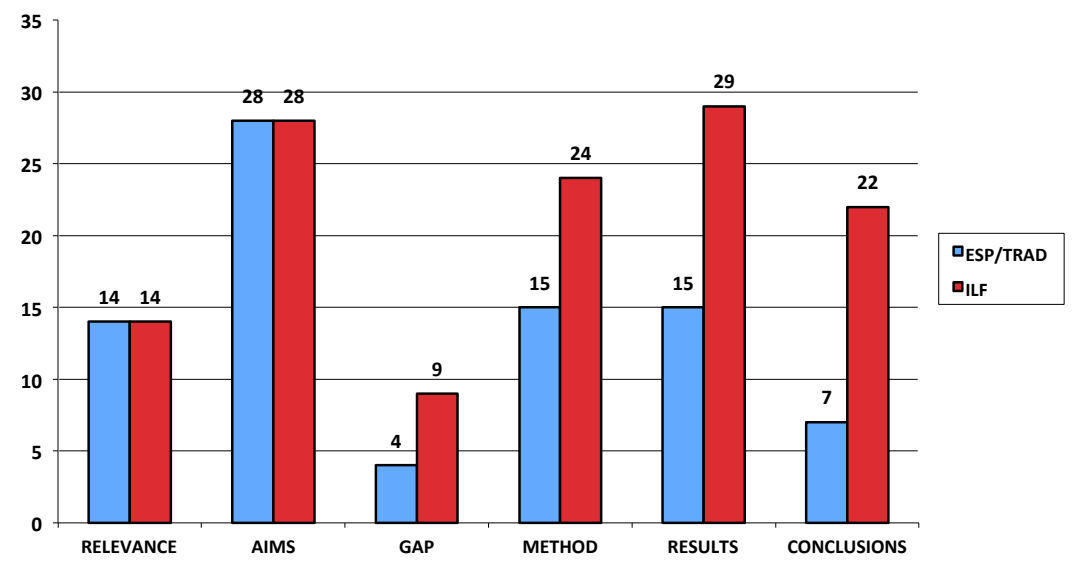

Figura 1. Presencia de movimientos en los 30 abstracts de Sociología en los subcorpus ESP/TRAD e ILF

Se observa una presencia similar de los movimientos Relevance y Aims en los subcorpus ESP/TRAD e ILF; Relevance aparece en 14 casos (46,7 \%) de los abstracts de los tres subcorpus, y Aims en la práctica totalidad (28 casos, lo que representa un 93,3\%). Por el contrario, Gap es el movimiento con menos presencia, especialmente en ESP/TRAD (13,3 \%, frente a un 30,0 \% en el subcorpus ILF). Los movimientos Method, Results y Conclusions tienen una menor frecuencia también en ESP/TRAD (50,0\%, 50,0 \% y 23,3\%, respectivamente), y mucho mayor en el subcorpus ILF (80,0 \%, 96,7 \% y 73,3\%). Puede destacarse la baja frecuencia de uso del movimiento Conclusions en ESP/TRAD. La distribución de los resultados en los subcorpus ESP/TRAD frente al subcorpus ILF es estadísticamente significativa (chi-cuadrado: 11,284; grados de libertad: 5, p-valor: 0,047).

Los resúmenes traducidos compiten en las bases de datos internacionales con un importante número de abstracts en ILF y una gran mayoría de abstracts en inglés L1 (lengua nativa) que suelen incluir dos de los movimientos más importantes: el movimiento Gap (localizar el nicho en el que contribuye el estudio) y las Conclusiones (qué es lo que aporta el estudio). Así, en un estudio previo sobre abstracts de Sociología en inglés L1, el Gap apareció en el $50 \%$ de los abstracts (Lorés-Sanz 2014).

La ausencia de Gap en los subcorpus ESP/TRAD se puede interpretar en la misma línea de otros estudios de textos académicos en español en los que tiende a evitarse la crítica, por ejem- 
plo, las reseñas de libros (Lorés-Sanz 2009b). Por otra parte, la falta de conclusiones parece entroncar con la tradición española de redacción de textos (y posiblemente con su enseñanza), que se constata en los artículos de investigación en secciones de resultados de gran extensión, y de discusiones muy breves (Mur-Dueñas 2007; Moreno 2010; Moreno et alii 2012).

\subsection{Fraseología}

En este apartado nos centramos en la comparación de la fraseología de los abstracts en inglés en los subcorpus TRAD e ILF, en relación con los distintos movimientos.

En el movimiento Relevance, con la misma frecuencia en los dos subcorpus (46,7 \%), no se encuentra ningún patrón que se repita en los abstracts analizados. La fraseología parece depender del tema de investigación, que es resaltado, o de un área problemática del mismo, sobre la que recae el foco, como podemos observar en los ejemplos (1)-(3):

(1) Class differences in educational decision making are important for inequality. [ILF 1]

(2) Survey methodologists are concerned that the interviewers characteristics may affect respondents answers. [ILF 24]

(3) In the same way as any other type of social organization, NGOs evidence the existence of gender inequalities in their structure and internal functioning. [TRAD 15]

En el movimiento Aims, en cambio, se repiten ciertos patrones que pueden observarse en la Tabla 1:

\begin{tabular}{|l|l|l|}
\hline & ILF & TRAD \\
\hline $\begin{array}{l}\text { Desagentivación o abstract rhetor (this paper) + verbo en voz activa (proceso } \\
\text { mental/cognitivo) }\end{array}$ & 16 & 13 \\
\hline $\begin{array}{l}\text { Marcador endofórico (in this article) + automención (I/we/the authors) + verbo en } \\
\text { voz activa (proceso mental/cognitivo) }\end{array}$ & 4 & 7 \\
\hline Automención (I/we/the authors) + verbo en voz activa (proceso mental/cognitivo) & 5 & --- \\
\hline Marcador de marco para anunciar el propósito (the purpose/aim is) & --- & 5 \\
\hline Verbo en voz pasiva & 3 & 3 \\
\hline
\end{tabular}

Tabla 1. Patrones fraseológicos del Move 2 Aims en los subcorpus en inglés ILF y TRAD

El patrón más común es la combinación de desagentivaciones (abstract rhetors) con verbos en voz activa de procesos mentales o cognitivos (por ejemplo, This article presents), especialmente en el subcorpus ILF. Asimismo, las automenciones (self-mentions) son relativamente frecuentes en el subcorpus ILF, con varios casos con un marcador endofórico (endophoric marker) (p. ej. In this article, I offer) o sin él (We analyze). En cambio, en el subcorpus TRAD, se encontraron también algunos casos, pero todos ellos sin marcadores endofóricos. Otra diferencia es el uso en el subcorpus TRAD de marcadores de marco (frame markers) para anunciar el propósito (The purpose of this article is to), dado que este patrón no se encontró en el subcorpus ILF. 
Estas categorías pueden ilustrarse mediante los siguientes ejemplos de los dos subcorpus, con secuencias completas:

- Desagentivación + verbo en voz activa:

(4) This study uncovers an unexpected effect of family-friendly policies on women's economic attainments. [ILF 6]

(5) This article presents an analytical proposal and its application by a case study. [TRAD 6]

- Marcador endofórico + automención + verbo en voz activa:

(6) In this article, I offer an alternative reading by supplementing a critical reading of the report with an analysis of its historical and immediate institutional context. [ILF 14]

(7) In this paper we look for similarities and differences regarding their use of concepts and expected results. [TRAD 28]

- Automención + verbo en voz activa:

(8) We analyze intra-family support among Palestinians living in Lebanon, (...) [ILF 30]

- Marcador de marco:

(9) The purpose of this article is to identify the most prominent features of a family policy model in Southern European countries. [TRAD 5]

- Verbo en voz pasiva:

(10) Concepts are discussed in relation to Chinese culture, as well as attachment and needs theories. [ILF 21]

(11) The feelings (opinion and attitudes) towards immigration in November 2005 are analysed in this study, (...) [TRAD 26]

Los resultados del tercer movimiento, Gap, se muestran en la Tabla 2:

\begin{tabular}{|l|c|c|}
\hline & ILF & TRAD \\
\hline Uso de conjunciones, marcadores discursivos o adverbios oracionales & 3 & 4 \\
\hline Uso de indicadores léxicos del nicho o gap & 6 & --- \\
\hline
\end{tabular}

Tabla 2. Patrones fraseológicos del Move 3 Gap en los subcorpus en inglés ILF y TRAD

En este movimiento se encontró un uso similar de conjunciones, marcadores discursivos y adverbios oracionales en ambos subcorpus. Sin embargo, en el corpus ILF se detectaron varios casos de indicadores léxicos, ausentes por completo en el corpus TRAD. Esta falta de indicadores léxicos en el Gap es consistente con la menor frecuencia de uso de este movimiento (ver sección 3.1.). En los ejemplos (12)-(14) se ilustran estos patrones. 
- Conjunciones, marcadores discursivos o adverbios oracionales:

(12) Nevertheless, we are not content with the claim of Preference Theory that this shift in commitment is merely a matter of not-so-dedicated women discovering their genuine preferences. [ILF 13]

(13) Although there are great differences for a consensual social capital definition, it is important to look for empirical indicators for its measurement. [TRAD 4]

- Indicadores léxicos:

(14) Several researchers have expressed doubts as to whether ANOVA is fit to detect interaction in a fixed $2 \times 2$ design. [ILF 28]

En la Tabla 3 podemos encontrar los datos de los patrones del movimiento Method, con algunas marcadas diferencias entre los dos subcorpus:

\begin{tabular}{|l|c|c|}
\hline & ILF & TRAD \\
\hline $\begin{array}{l}\text { Indicador semántico: sintagma preposicional (by means of/through the use of, } \\
\text { thanks to) }\end{array}$ & 1 & 2 \\
\hline Indicador semántico: gerundio (using, drawing on) + noun & 11 & 1 \\
\hline Automención (we exclusivo) + verbo en voz activa (use) & 1 & 8 \\
\hline Verbo en voz pasiva & 7 & 3 \\
\hline Desagentivación + verbo epistémico (the study/this research) & 3 & 1 \\
\hline Sin indicador & 1 & --- \\
\hline
\end{tabular}

Tabla 3. Patrones fraseológicos del Move 4 Method en los subcorpus en inglés ILF y TRAD

En el subcorpus ILF, los patrones más frecuentes fueron los indicadores semánticos, en particular gerundios como using. Los verbos en voz pasiva también fueron bastante comunes, siguiendo las convenciones de este movimiento. Por el contrario, en el subcorpus TRAD, las automenciones fueron el patrón más frecuente. Esta tendencia reflejaría una transferencia de la retórica del español, dado que las automenciones se usan en movimientos que conllevan una menor amenaza para la imagen de los autores (cf. Lorés-Sanz 2011). Se dieron también algunos casos ocasionales de otros patrones como sintagmas preposicionales y desagentivaciones, en los dos subcorpus. Los siguientes ejemplos incluyen estos patrones:

- Indicadores semánticos (sintagmas preposicionales):

(15) Thanks to common foundations in both epistemology and ontology, (...) [ILF 7]

(16) (...) through the study of the practices, dynamics and rhetoric that go on inside them. [TRAD 15] 
- Indicadores semánticos (gerundio + nombre):

(17) Drawing on employment history data from the Survey of Income and Program Participation and the German Socio-Economic Panel, (...) [ILF 10]

(18) (...) using a qualitative methodology for the analysis of the information (...) [TRAD 29]

- Automención + verbo en voz activa:

(19) I evaluate the Stasi Report in its immediate context of institutional change, (...) [ILF 14]

(20) We have centred our field work on the prisons of Huelva, Algeciras (Cádiz) and Alhaurín de la Torre (Málaga). [TRAD 22]

- Voz pasiva:

(21) Register-based data and multi-level statistical analysis are used to assess the existence and form of neighborhood effects on the educational careers of young people in Helsinki. [ILF 27]

(22) A survey carried out by the CIS (2005, study no. 2590) on 2,490 citizens from a representative sample of the Spanish population was used. [TRAD 12]

- Desagentivación + verbo epistémico:

(23) The data include register information on all pupils who attended the ninth grade of the Swedish compulsory school in 1998 and 1999 and their parents $(n=73,824)$. [ILF 15]

(24) It [the article] takes as an example the discursive oscillations and contradictions on weight control of working class mothers. [TRAD 23]

Con respecto al movimiento Results, se aprecia mayor variedad de patrones en el corpus ILF, como puede verse en la Tabla 4:

\begin{tabular}{|l|c|c|}
\hline & ILF & TRAD \\
\hline Desagentivación (the paper/the analysis) + verbo epistémico (demonstrates/argues) & 18 & 10 \\
\hline Automención (I/we/the author(s)) + verbo en voz activa (verbo epistémico) & 5 & --- \\
\hline Verbo en voz pasiva & 2 & 5 \\
\hline Sin indicador & 4 & --- \\
\hline
\end{tabular}

Tabla 4. Patrones fraseológicos del Move 5 Results en los subcorpus en inglés ILF y TRAD

En este movimiento, las desagentivaciones fueron los indicadores léxico-gramaticales más frecuentes en los dos subcorpus, especialmente en el corpus ILF. En este subcorpus encontramos también varias automenciones y algunos casos de ausencia de indicadores y de verbos en voz pasiva. Sin embargo, en el subcorpus TRAD se da una notable ausencia de automenciones para resaltar los resultados de la investigación, mientras que la voz pasiva tiene cierta presencia. De nuevo, nos encontramos con una posible transferencia de la retórica del español, dado que este movimiento de resultados conlleva cierta carga impositiva hacia el lector, y por ello se tiende a la no presencia explícita de los autores (cf. Lorés-Sanz 2011). 
- Desagentivación + verbo epistémico:

(25) The analysis shows that gender earnings disparities are less pronounced in countries with developed family policies. [ILF A6]

(26) This paper shows that autoregulation of violence is necessary but imposible [sic] in the State Nation frame. [TRAD 3]

- Automención + verbo en voz activa (mental/epistémico):

(27) We find that cross-national patterns differ from those found in previous studies. [ILF 23]

- Voz pasiva:

(28) It will be shown that immigrants have indeed higher turnover rates, (...) [ILF 5]

(29) While a low rate of female employment is probably associated with a lack of familyfriendly provisions, the model is reproduced because care services within the family are not externalized to the market. [TRAD A5]

- Sin indicador:

(30) Individuals who give a high social value to the innovation tend to look for information that allows them to evaluate more precisely the individual benefit of adoption. If the social value they assign is low, they neither consider the information nor transmit it. [ILF 8]

Finalmente, en la Tabla 5 pueden observarse los resultados del movimiento Conclusions:

\begin{tabular}{|l|c|c|}
\hline & ILF & TRAD \\
\hline $\begin{array}{l}\text { Desagentivación (this conclusion/these findings + verbo epistémico (suggest(s), } \\
\text { contribute(s), imply) }\end{array}$ & 13 & 5 \\
\hline Automención (I/we/the author(s)) + verbo en voz activa (mental/epistémico) & 4 & 1 \\
\hline Verbo en voz pasiva & 2 & 1 \\
\hline Marcadores discursivos, adverbios oracionales (as a result, thus) & 3 & --- \\
\hline
\end{tabular}

Tabla 5. Patrones fraseológicos del Move 6 Conclusions en los subcorpus en inglés ILF y TRAD

En este movimiento las principales diferencias radican en el uso más frecuente de automenciones y desagentivaciones en el corpus ILF. Otros patrones encontrados fueron el uso de conectores y de verbos en voz pasiva, aunque no apareció ningún caso de estos últimos en el corpus TRAD. Los ejemplos (31)-(38) muestran estas realizaciones:

- Desagentivación + verbo epistémico:

(31) This article contends that syndicating endured because of (...) [ILF 5]

(32) As well as this paper shows the utility of social capital concept as a quality life predictor. [TRAD 4] 
- Automención + verbo en voz activa (mental/epistémico):

(33) The author argues that the ethical-affective principle that defines strong ties (...) [ILF 2]

(34) (...) and [we] conclude that no single theory may address all collective action problems (...) [TRAD 28]

- Voz pasiva:

(35) The value differences between the educational groups should thus not be seen as reflecting economic differences between the groups but ... [ILF 16]

(36) It was also found how the differences in social perception of the behaviour of Spaniards on environmental matters are associated with ... [TRAD 12]

- Conectores, adverbios oracionales:

(37) It was also found how the differences in social perception of the behaviour of Spaniards on environmental matters are associated with (...) [TRAD 12]

(38) As a result South Korean women have experienced dramatic changes in marriage patterns, fertility, family relations, etc. [ILF 11]

Por último, en los distintos movimientos de los abstracts del subcorpus TRAD se dan estructuras que son un claro reflejo de los patrones de uso del español, constituyendo casos de calco. Por ejemplo: This paper is derived (...), como traducción de El presente artículo deriva de (...); The article defends (...), por El articulo defiende (...); o At this article, $X$ are investigated, por En el presente artículo se indagan $X$.

\section{CONCLUSIONES}

En este artículo hemos identificado las diferencias en la estructura retórica y los patrones fraseológicos en dos subcorpus de abstracts de Sociología escritos en inglés como lengua franca (subcorpus ILF) y en inglés como lengua traducida del español (subcorpus TRAD, cuyos textos reflejan de forma consistente la estructura de sus textos origen del corpus de abstracts escritos en español [subcorpus ESP]). En relación con la estructura, se aprecian similitudes en los movimientos de Relevance y Aims. Por otra parte, las principales diferencias se derivan de la frecuente ausencia de los movimientos Gap y Conclusions en el subcorpus de traducciones al inglés. Estas diferencias reflejan la retórica propia de los trabajos de investigación en español, es decir, de la retórica académica (Mur-Dueñas 2007; Lorés-Sanz 2009b; Moreno 2010; Moreno et alii 2012).

Por lo que se refiere a los patrones fraseológicos, nos encontramos también con semejanzas en los subcorpus TRAD e ILF, como el predominio de estructuras con agentes no personales. Las diferencias constatadas se dan en el uso de desagentivaciones o abstract rhetors, con mayor frecuencia en el subcorpus ILF. Además, el subcorpus TRAD muestra una mayor frecuencia de agentes no personales en los movimientos Aims y Results y de automenciones en el movimiento de Method, en contra de las convenciones habituales de los abstracts en inglés L1. Las automenciones se dan, pues, en movimientos que suponen una menor amenaza para la imagen de los autores, frente al uso de la voz pasiva cuando 
el movimiento retórico implica algún tipo de imposición hacia el lector, y todo ello puede suponer una transferencia de la retórica del español (cf. Lorés-Sanz 2011). Por otra parte, en el subcorpus TRAD aparecen estructuras que son un reflejo claro de patrones de uso del español. Esta tendencia en ocasiones da lugar a calcos que convendría quizás evitar.

Las limitaciones del estudio residen quizás en el limitado número de abstracts del subcorpus y en la variedad de los patrones fraseológicos detectados, la cual no nos ha permitido ir mucho más allá de un estudio cuantitativo exploratorio. No obstante, sí que ha podido obtenerse suficiente información para sustentar un estudio cuantitativo de la estructura retórica de los abstracts y para llevar a cabo un análisis cualitativo de los patrones fraseológicos, así como para apreciar diversas tendencias en el análisis cuantitativo de estos últimos.

Desde nuestro punto de vista, este estudio supone una novedad en tanto en que utiliza como término de comparación textos escritos en inglés por investigadores cuya primera lengua no lo es (subcorpus ILF). Es decir, adopta como referencia el inglés como lengua franca (Seidlhofer 2011; Mauranen 2012), y no el inglés L1, como se ha venido haciendo hasta ahora. Las implicaciones pueden ser múltiples; sin embargo, los estudios con textos en ILF pueden representar un foco de estudio más realista, dado que estos textos suponen un objetivo más asequible para los investigadores españoles y para los investigadores no nativos en general, en términos de retórica y patrones léxico-gramaticales.

\section{Referencias bibliográficas}

Alharbi, L. (1997). "Rhetorical Transfer across Cultures: English into Arabic and Arabic into English", Journal of Applied Linguistics, 11(2), pp. 69-94.

Alharbi, L. y J. M. Swales (2011). "Arabic and English Abstracts in Bilingual Language Science Journals: Same or Different?", Languages in Contrast, 11(1), pp. 69-85.

Alonso-Almeida, F. (2014). "Evidential and Epistemic devices in English and Spanish Medical, Computing and Legal Scientific Abstracts: A Contrastive Study". En Bondi, M. y R. Lorés-Sanz (eds.), pp. 21-42.

Bellés-Fortuño, B. y M. Querol-Julián (2010). "Evaluation in Research Article Abstracts: A Crosscultural Study between Spanish and English Medical Discourse”. En Lorés-Sanz, R., P. Mur-Dueñas y E. Lafuente-Millán, (eds.), pp. 83-98.

Bennett, K. (ed.) (2014). The semiperiphery of academic writing. Londres: Palgrave MacMillan.

Bondi, M. y R. Lorés-Sanz (eds.) (2014). Abstracts in Academic Discourse: Variation and Change. Berna: Peter Lang.

Burgess, S. y P. Martín-Martín (2010). "Interpersonal Features of Spanish Social Sciences Journal Abstracts”. En Lorés-Sanz, R., P. Mur-Dueñas y E. Lafuente-Millán, (eds.), pp. 99-115.

Busch-Lauer, I. A. (1995). "Textual organization in English and German abstracts", Anglicana Turkuensia, 14 , pp. 175-186.

Busch-Lauer, I. A. (2014). "Abstracts: Cross-linguistic, disciplinary and intercultural perspectives". En Bondi, M. y R. Lorés-Sanz (eds.), pp. 43-63.

Canagarajah, S. (1996). "Nondiscursive requirements in academic publishing, material resources of periphery scholars, and the politics of knowledge production", Written Communication, 13(4), pp. 435-472.

Canagarajah, S. (2002). A geopolitics of academic writing. Pittsburgh: University of Pittsburgh Press.

Carey, R. (2013). "On the other side: Formulaic organizing chunks in spoken and written academic ELF", Journal of English as a Lingua Franca, 2(2), pp. 207-228.

Cogo, A. y M. Dewey (2012). Analysing English as a lingua franca: A corpus-driven investigation. Londres: Continuum. 
Diani, G. (2014). “On English and Italian Research Article Abstracts”. En Bondi, M. y R. Lorés-Sanz (eds.), pp. 65-83.

Divasson, L. e I. K. León Pérez (2006). "Textual and Language Flaws: Problems for Spanish Doctors in Producing Abstracts in English", Ibérica, 11, pp. 61-79.

Ferguson, G. (2007). "The global spread of English, scientific communication and ESP: questions of equity, access and domain loss", Ibérica, 13, pp. 7-38.

Fernández Antolín, M. J., B. López Arroyo y R. de Felipe Boto (2006). "Contrasting the Rhetoric of Abstracts", Hermēneus. Revista de Traducción e Interpretación, 8, pp. 85-110.

Flowerdew, J. (2007). "The non-Anglophone scholar on the periphery of scholarly publication", AILA Review, 20, pp. 14-27.

Flowerdew, J. (2008). "Scholarly writers who use English as an additional language: What can Goffmans Stigma tell us?", Journal of English for Academic Purposes, 7(2), pp. 77-86.

Granger, S. (2003). "The Corpus Approach: A Common Way forward for Contrastive Linguistics and Translation Studies". En Granger, S., J. Lerot y S. Petch-Tyson (eds.). Corpus-based Approaches to Contrastive Linguistics and Translation Studies. Amsterdam y Atlanta: Rodopi, pp. 17-29.

House, J. (2009). "Introduction. The pragmatics of English as a lingua franca", Intercultural Pragmatics, $6(2)$, pp. 141-145.

Işık-Taş, E. E. (2018). "Authorial identity in Turkish language and English language research articles in Sociology: The role of publication context in academic writers' discourse choices", English for Specific Purposes, 49, pp. 26-38.

Jenkins, J. (2000). The Phonology of English as an International Language. Oxford: OUP.

Kaplan, R. K. (1966). "Cultural Thought Patterns in Inter-cultural Education", Language Learning, 16(1-2), pp. 1-20.

Lafuente-Millán, E. (en prensa). "Evaluation in research article introductions in the Social Sciences written by ENL and ELF users". En Mur-Dueñas, P. y J. Sinkuniene (eds.).

Lewin, B. A. (2010). Writing Readable Research. Londres: Equinox.

Lillis, T. y M. J. Curry (2010). Academic writing in a global context. The politics and practices of publishing in English. Londres: Routledge.

López Arroyo, B., M. J. Fernández Antolín y R. de Felipe Boto (2007). "Contrasting the Rhetoric of Abstracts in Medical Discourse: Implications and Applications for English Spanish Translation", Languages in Contrast, 7(1), pp. 1-28.

Lorés-Sanz, R. (2006). "I Will Argue that: First Person Pronouns and Metadiscoursal Devices in RA Abstracts in English and Spanish", ESP across Cultures, 3, pp. 23-40.

Lorés-Sanz, R. (2009a). "Different Worlds, Different Audiences: A Contrastive Analysis of Research Article Abstracts". En Suomela-Salmi, E. y F. Dervin, (eds.). Cross-Linguistic and Cross-Cultural Perspectives on Academic Discourse. Amsterdam/Philadelphia: John Benjamins, pp. 187-198.

Lorés-Sanz, R. (2009b). "(Non-)Critical Voices in the Reviewing of History Discourse: A Cross-Cultural Study of Evaluation”. En Hyland, K. y G. Diani (eds.). Academic Evaluation: Review Genres in University Settings. Londres: Palgrave Macmillan, pp. 143-160.

Lorés-Sanz, R. (2011). "The construction of the author's voice in academic writing: the interplay of cultural and disciplinary factors", Text \& Talk, 31(2), pp. 173-193.

Lorés-Sanz, R. (2014). "Lost (and Gained) in Translation: A Contrastive (English/Spanish) Analysis of Rhetorical and Lexicogrammatical Patterns in Sociology RA Abstracts”. En Bondi, M. y R. LorésSanz (eds.), pp. 85-109.

Lorés-Sanz, R. (2016). "ELF in the making?: Simplification and hybridity in abstract writing", Journal of English as a Lingua Franca, 5(1), pp. 53-81.

Lorés-Sanz, R. (2018a). "The Use of ELF in International Online Conference Announcements: Changing Modes and Means of Academic Communication". English Text Construction, 11(2), pp. 256-284.

Lorés-Sanz, R. (2018b). "Online conference announcements as spaces for disciplinary communication". En Vázquez-Orta, I. e I. Guillén-Galve (eds.), pp. 237-272. 
Lorés-Sanz, R. (en prensa). "Hybrid rhetorical structure in English Sociology research article abstracts: The ambit of ELF and translation”. En Mur-Dueñas, P. y J. Sinkuniene (eds.).

Lorés-Sanz, R., P. Mur-Dueñas y E. Lafuente-Millán (eds.) (2010). Constructing Interpersonality. Multiple Perspectives on Written Academic Genres. Newcastle upon Tyne: Cambridge Scholars Publishing.

Martín-Martín, P. (2003). "A Genre Analysis of English and Spanish Research Paper Abstracts in Experimental Social Sciences", English for Specific Purposes, 22(1), pp. 25-43.

Martín-Martín, P. (2005). The Rhetoric of the Abstract in English and Spanish Scientific Discourse. A Cross-Cultural Genre-Analytic Approach. Berna: Peter Lang.

Mauranen, A. (2009). "Chunking in ELF: Expressions for managing interaction", Journal of Intercultural Pragmatics, 6(2), pp. 217-233.

Mauranen, A, (2012). Exploring ELF: Academic English shaped by non-native speakers. Cambridge: Cambridge University Press.

Moreno, A. I. (2010). "Researching into English for research publication purposes from an applied intercultural perspective". En Ruiz-Garrido, M. F., J. C. Palmer-Silveira e I. Fortanet-Gómez (eds.). English for Professional and Academic Purposes. Amsterdam: Rodopi, pp. 57-71.

Moreno, A. I., J. Rey-Rocha, S. Burgess, I. López-Navarro e I. Sachdev (2012). "Spanish researchers' perceived difficulty writing research articles for English medium journals: the impact of proficiency in English versus publication experience", Ibérica, 24, pp. 157-184.

Mur-Dueñas, P. (2007). "A cross-cultural analysis of the research article discussion move". En Mairal, R. et alii (ed.). Aprendizaje de lenguas, uso del lenguaje y modelación cognitiva: perspectivas aplicadas entre disciplinas. AESLA y UNED, pp. 1259-1267.

Mur-Dueñas, P. (2013). "Spanish scholars research article publishing process in English medium journals: English used as a lingua franca?", Journal of English as a Lingua Franca, 2(2), pp. 315-340.

Mur-Dueñas, P. (2015). "Looking into ELF variants: A study of evaluative it-clauses in research articles", ESP Today, 3(2), pp. 160-179.

Mur-Dueñas, P. (2016). "Modal hedging verbs in English-medium research articles: A look into written academic ELF", Kalbotyra, 69, pp. 152-177.

Mur-Dueñas, P. (2018). "Exploring interpersonality features in ELF research articles: from rhetorical and discursive homogeneity to lexico-grammatical hybridity". En Vázquez-Orta, I. e I. Guillén-Galve (eds.), pp. 209-233.

Mur-Dueñas, P. (en prensa). "Exploring ELF manuscripts: An analysis of the anticipatory it-pattern with an interpersonal function". En Mur-Dueñas, P. y J. Sinkuniene (eds.).

Mur-Dueñas, P. y J. Sinkuniene (eds.) (en prensa). Intercultural perspectives on research writing. Amsterdam: John Benjamins.

Murillo, S. (2018). "Discourse markers and academic writing in English as a Lingua Franca". En Vázquez-Orta, I. e I. Guillén-Galve (eds.), pp. 151-177.

Murillo, S. (en prensa). "Not the same, but how different? Contrasting the use of reformulation markers in ELF and ENL research articles". En Mur-Dueñas, P. y J. Sinkuniene (eds.).

Perales-Escudero, M. y J. M. Swales (2011). "Tracing Convergence and Divergence in Pairs of Spanish and English Research Article Abstracts: The case of Ibérica", Ibérica, 21, pp. 49-70.

Pho, P. D. (2008). "Research article abstracts in applied linguistics and educational technology: a study of linguistic realizations of rhetorical structure and authorial stance", Discourse Studies, 10(2), pp. 231-250.

Preacher, K. J. (2001). En línea. Calculation for the chi-square test: An interactive calculation tool for chi-square tests of goodness of fit and independence [Software]. http://www.quantpsy.org (1/3/2018).

Seidlhofer, B. (2012). "Anglophone-centric attitudes and the globalization of English", Journal of English as a Lingua Franca, 1(2), pp. 393-407.

Tardy, C. (2004). "The role of English in scientific communication: Lingua franca or tyrannosaurus rex?", Journal of English for Academic Purposes, 3(3), pp. 247-269. 
Tognini-Bonelli, E. (2001). Corpus Linguistics at Work. Amsterdam: John Benjamins.

Van Bonn, S. y J. M. Swales (2007). "English and French Journal Abstracts in the Language Sciences: Three Exploratory Studies", Journal of English for Academic Purposes, 6(2), pp. 93-108.

Vázquez-Orta, I. e I. Guillén-Galve (eds.) (2018). ELF and Intercultural Communication: implications and/or applications to the field of English as Foreign Language. Berlin: Peter Lang.

\section{APÉNDICE 1 - Normas para autores}

Las seis revistas que se han utilizado para la compilación del corpus incluyen instrucciones sobre la redacción de los abstracts o resúmenes de artículos de investigación. Estas instrucciones pueden resultar de interés para contextualizar el estudio que aquí se presenta.

A) Revistas españolas:

Papers. Revista de Sociología

El texto de los artículos y notas irá precedido de un resumen de no más de 250 palabras (que expondrá clara y concisamente los objetivos, metodología, principales resultados y conclusiones del trabajo).

Revista Española de Investigaciones Sociológicas (REIS)

Los trabajos irán precedidos de un breve resumen de entre 100 y 130 palabras, que tendrá una versión en español y otra en inglés, siendo ambas de idéntico contenido. El resumen debe exponer con claridad la finalidad del estudio o investigación (objetivos); los procedimientos básicos (métodos); los principales hallazgos (resultados), y las conclusiones más relevantes, así como resaltar los aspectos nuevos e importantes del estudio.

Revista Internacional de Sociología (RIS)

Es imprescindible que cada trabajo conste de un resumen de un máximo de 150 palabras tanto en español como en inglés así como de un máximo de cinco palabras clave, no incluidas en el título, igualmente en español y en inglés.

A) Revistas internacionales en inglés:

British Journal of Sociology

An abstract of up to 300 words, giving a concise statement of the intention, results and conclusions of the paper should be attached to the article.

AJS (American Journal of Sociology)

Your abstract should be as close to 100 words as possible. It should include your research question or puzzle, identify your data, and give some indication of your findings. Your abstract is likely to be sent by email to potential readers: giving an accurate and efficient statement of your project is likely to increase your chances of enlisting their aid. Unfocused, verbose abstracts may make it harder to place your paper with referees. Papers without an abstract cannot be sent to readers for peer review.

\section{Social Science Research}

A concise and factual abstract is required. The abstract should state briefly the purpose of the research, the principal results and major conclusions. An abstract is often presented separately from the article, so it must be able to stand alone. For this reason, references should be avoided, but if essential, then cite the author(s) and year(s). Also, non-standard or uncommon abbreviations should be avoided, but if essential they must be defined at their first mention in the abstract itself. 